\title{
Compact Wide Stop-Band Dual-Mode Filter with Four Transmission Zeros
}

\author{
Xue Bing ${ }^{1,2 *}$, Ji Yicai ${ }^{1,2}$ \\ 1 Institute of Electronics, Chinese Academy of Sciences, Beijing, China. \\ ${ }^{2}$ Key Laboratory of Electromagnetic Radiation and Sensing Technology, Chinese Academy of Sciences, Beijing, \\ China. \\ * Corresponding author. Tel: 086-010-58887497; email: xuebing14@mails.ucas.ac.cn \\ Manuscript submitted February 21, 2016; accepted June 12, 2016. \\ doi: 10.17706/ijcee.2016.8.3.234-240
}

\begin{abstract}
The size and the isolation of filters impact directly the whole front-end system integration and performance. The miniaturization and high-isolation of filters is always a hot spot of research. In this paper, a compact high-isolation band-pass filter is designed based on the dual mode short-ended resonator. The technique of the stepped-impedance lines can adjust the stop-band width by the length and width of the lines. In the designing process, the length of the feeders computed in an easy and effective way is given to provide the estimated value. The capacitive source-load coupling provides four transmission zeros to enhance the selectivity. The area of this filter, of which the chamfer micro-strips are used to decrease the size, is only $36.7 \%$ of the traditional ones. In addition, the characteristics of this filter are analyzed, from which the design graph is given and the measured results match well with the simulations.
\end{abstract}

Key words: Dual-mode filter, short-ended, compact, stepped- impedance lines.

\section{Introduction}

Microwave filter is the one of the most important components in the microwave and millimeter-wave system. It is widely used in the wireless communication, especially for the radar system. The main technical parameters of the filter include the pass-band selectivity, the harmonic suppression of the stop band, the bandwidth, the volume and the low insertion loss. The miniaturization and high performance of microwave filters is always a hot research topic [1], [2].

In recent years, the dual-mode filter based on microstrip provides a chance for the researchers. So the researchers and engineers prefer to study and apply this kind of filter because of its advantages. Compared with the traditional filter, stub-loaded dual-mode filter with tunable pass-band, miniaturization, and easy integration is a very useful component. There are many inventions of a variety of the dual-mode filters in recent papers. [3] gives a open-loop structure. The structure of the open-ended resonator decrease the size of filter. [4] reports a closed loop structure, which can reduce the number of the earthing compared with the same style filter. So, it enables the miniaturization of the whole filter and makes the integration easier. However, this filter has the short-ended resonator, which makes the size bigger than the one with the open-ended resonator.

Besides, for improving the performance, the transmission zeros are researched to improve the selectivity of the pass-band. Many papers report the effects of the numbers and the locations of the transmission zeros 
and how to control them [5]-[7]. [5] gives the relationship between the transmission zero location and the resonant frequency: when $f_{\text {odd }}>f_{0}$ and $f_{\text {even }}<f_{0}$, the transmission zero is at the lower stop-band; when $f_{\text {odd }}<f_{0}$ and $f_{\text {even }}>f_{0}$, the transmission zero is at the upper stop-band ( $f_{0}$ is the center frequency). It is useful to change the location of transmission zero by turning the length of loaded open-circuited-stub. [6] shows a dual-mode filter with a triangular structure that is a compact structure and the relationship between the zero location and the resonance frequency as [5], which can be obtained by changing the length of the inductive coupling lines. But the isolation of the filters in [5], [6] is not high. [4] reports a method for the enhancement of source-load coupling. By adding the open-ended stub besides the coupling lines, this structure increases the transmission zeros to improve the selective performance of the filter and the properties of the pass-band. However, this kind of filter have a relatively large structure. [7] reports a hexagonal dual-mode filter with inductive-stub loaded; by adjusting parameters of inductive-stub to the filter, the filter can have four transmission zeros, which can improve the selectivity of the pass-band efficiently.

In addition, when the fundamental frequency is very low, location of the second harmonic frequency is especially closed to the fundamental frequency, which greatly affects the quality of the filter. Some articles give the methods to adjust the width of the stop-band. [8], [9] use the stepped-impedance lines to control the fundamental and the second harmonic frequency to realize the wide pass-band. By changing sizes of the stepped-impedance lines, the fundamental frequency can be moved to the lower frequency and the second harmonic-frequency is moved to the upper frequency.

Last, the dual mode resonators can be applied in many other components. [10]-[12] give an application about diplexers. Dual mode resonators in this kind of components give a wide-band to filter the frequency needed. [10] reports a dual mode short-ended resonator using in the diplexer that can distinguish signals into different bands of the UMTS and WCDMA. [11] uses the open-ended resonator to separate the different signals into GSM and WLAN bands, respectively. The open-ended resonators in [12] provide a high-isolation for the electromagnetic waves in the different bands.

Based on the design ideas and methods of the literatures [3]-[9], we make a L-band filter using the closed loop to obtain the wide stop-band using the stepped-impedance lines, which make the second harmonic frequency at the $4.1 f_{0}$. The open-ended stub besides the coupling lines provides this filter with four transmission zeros. The third zero can change with the length and the distance of this open-ended stub. Furthermore, the open-ended stub can give a high-isolation by controlling the first and the second zeros. For the smaller size, we make the low impedance line folded and give a resection, which forms a compact structure.

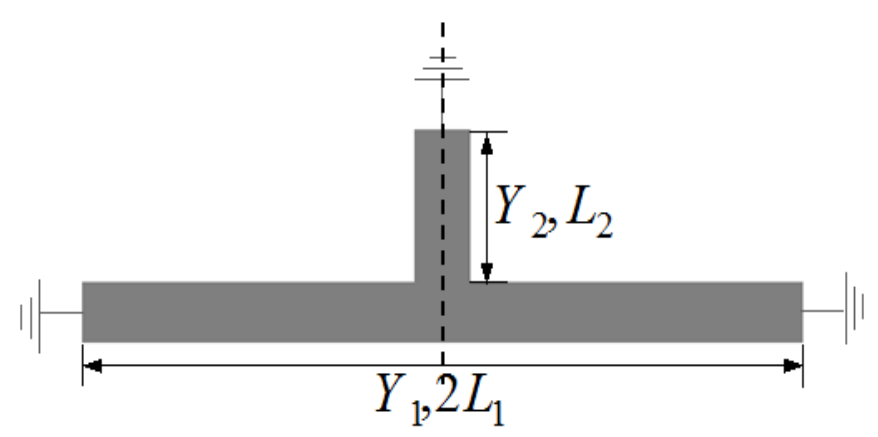

Fig. 1. Short-ended stub-loaded resonator.

\section{Filter Design}

\subsection{Stepped-Impedance Resonator}


Stub-loaded dual-mode filter has four fundamental resonant configurations, which are the short-ended stub loaded open-ended resonator [3], the short-ended stub loaded short-ended resonator [4], the open-ended stub loaded short-ended resonator, and the open-ended stub-loaded open-ended resonator [5], [6], respectively. This paper uses the short-ended stub loaded resonator shown in Fig. 1, which makes the both sides of pass-band have two transmission zeros.

Then, we use the model shown in Fig. 2 to analyze the odd and even mode. The $L_{1}$ is the length of the high impedance line, the $L_{2}$ is the length of the low impedance line, the $L_{3}$ is the length of the short-ended, the $Z_{1}$ and $Z_{2}$ are the impedance values. In the design of narrow-band filter, we can assume that the open-ended-stub is the perturbation of the resonator. When the effect of $L_{3}$ cannot be considered, according to the theory of the transmission line, the formula can be obtained by derivation:

$$
\begin{gathered}
A * \tan \left(K \theta_{L}\right)=\cot \left[(1-K) \theta_{L}\right] \\
A * \tan \left(K \theta_{L}\right)=-\tan \left[(1-K) \theta_{L}\right]
\end{gathered}
$$

where, $\theta_{L}=\theta_{L_{1}}+\theta_{L_{2}}, \quad A=\frac{Z_{1}}{Z_{2}}, \quad K=\frac{\theta_{L_{1}}}{\theta_{L}} . \theta$ represents the transmission line phase. These equations give the relationship between the parameters of the transmission lines. When the parameters meet the equations, the rate of the resonant frequencies can be obtained. We can compute the parameters of the transmission lines to meet the engineering requirements.

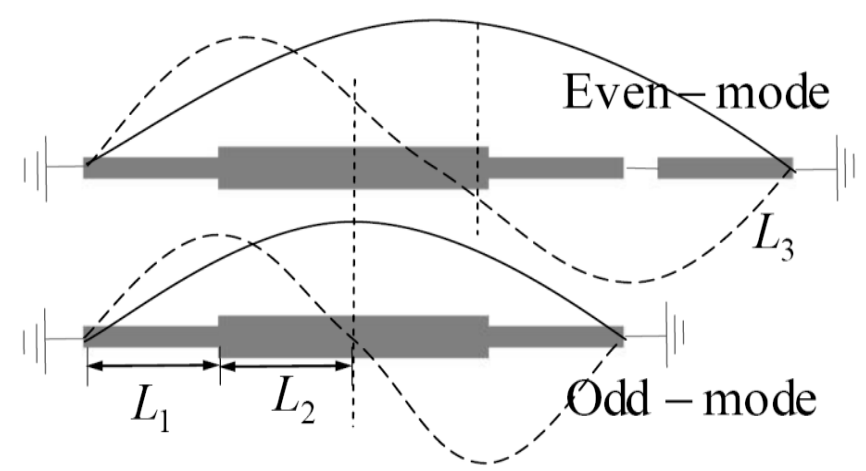

Fig. 2. Odd-mode and even-mode equivalent circuit of the stepped-impedance dual-mode resonator and the electrical field intensity distribution.

\subsection{The Source and the Load Lines Design}

The coupling line between the feeders and the resonator of filter is a very important component for the filter. Several factors should be considered for the coupling, such as the width of the feeders, the distance between the feeder and the resonators and the coupling lengths of the coupling lines. Now, we are going to analyze the effects of coupling length qualitatively. If the length of coupling line covers both the positive and the negative half-cycle of the wavelength in the resonant frequency, the feed can be offsetted for this kind of determined mode. On the contrary, when the length of coupling line only covers the positive or the negative half-cycle of the wavelength in resonant frequency, the feed is enhanced. As is shown in the Fig. 3, for the $f_{1}$ resonant frequency, the feed is in a large state. 
Due to the short-ended stub loaded short-ended resonator, the feed of the odd-mode and the even-mode of the fundamental frequencies are enhanced. Meanwhile, the feed of the second harmonic mode should be relatively destructive. So, for the feed method shown in Fig. 3, we can list four kinds of the relational equations, which can meet the best conditions of the feed: $L_{3}=L_{1} / 2, L_{3}=\left(L_{1}+L_{2}\right) / 2, L_{3}=L_{1}-L_{2}, L_{3}=L_{1}$. $L_{1}$ is the length of odd-mode resonator, $L_{2}$ is the length of short-ended stub, $L_{3}$ is the length of feeder.

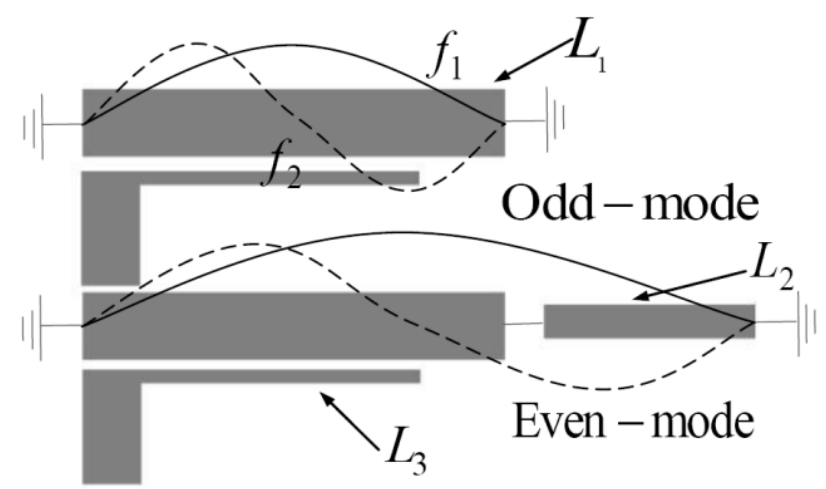

Fig. 3. The feed structure and field distribution of even-mode and odd-mode resonator.

Using the concept of weighted residuals, we set $U_{i}(i=1,2,3,4)$ as the residuals of four relational equations, to establish the Lagrange equation:

$$
f\left(L_{3}\right)=\sum_{i=1}^{4} k_{i}\left|U_{i}\right|
$$

When $f\left(L_{3}\right)$ is the smallest, the optimal solution can be obtained. Let $k_{i}=1$, for the filter as shown in Fig. 3 of [4]. When $L_{1}=24 \mathrm{~mm}$ and $L_{2}=3.1 \mathrm{~mm}$, we use this equation to get $L_{3}=13.6 \mathrm{~mm}$. Whereas $L_{3}$ in [4] is $13.9 \mathrm{~mm}$. For the filter we designed in this paper, using $L_{1}=51 \mathrm{~mm}$ and $L_{2}=1.5 \mathrm{mmcan}$ obtain $L_{3}=26.3 \mathrm{~mm}$, which is similar to the simulation design value of $L_{3}=27 \mathrm{~mm}$. So, we may use (3) to give the original values of the feeders for the simulation and the optimization.

\section{Simulation and Measurement}

According to the design method in Section 2 of this paper, a L-band narrow-band band-pass filter is designed as shown in Fig. 4, which consists of a closed loop structure and stepped-impedance lines. So as to reduce the external radiation and overall volume, the low impedance line is folded into $90^{\circ}$ and the corner is cut off. High-impedance lines are used as the feeders, and the auxiliary coupling line is added for source load coupling. By computing, the width of the $50 \Omega$ transmission line $\left(W_{0}\right)$ is determined as $2.7 \mathrm{~mm}$. Because of using $A=4.7$ and $K=0.64$ in Section 2 and setting the $W_{1}=0.2 \mathrm{~mm}$, we can get the $W_{3}=5 \mathrm{~mm}$ by the empirical formula of microstrip line. Then, $f_{2} / f_{0} \approx 4.1$ can be obtained. In this paper, the substrate relative dielectric constant is 2.65 and the substrate thickness is $1 \mathrm{~mm}$. Because of $\lambda_{g}=c_{g} / f_{0}$ and $c_{g}=c / \sqrt{\varepsilon_{\text {eff }}}$, when $f_{0}=1.3 \mathrm{GHz}, f_{2} \approx f_{0} * 4.1 \approx 5.3 \mathrm{GHz}$ can be obtained. So according to the simulation, the final size of the design: $L_{1}=18 \mathrm{~mm}, L_{2}=7.5 \mathrm{~mm}$. 


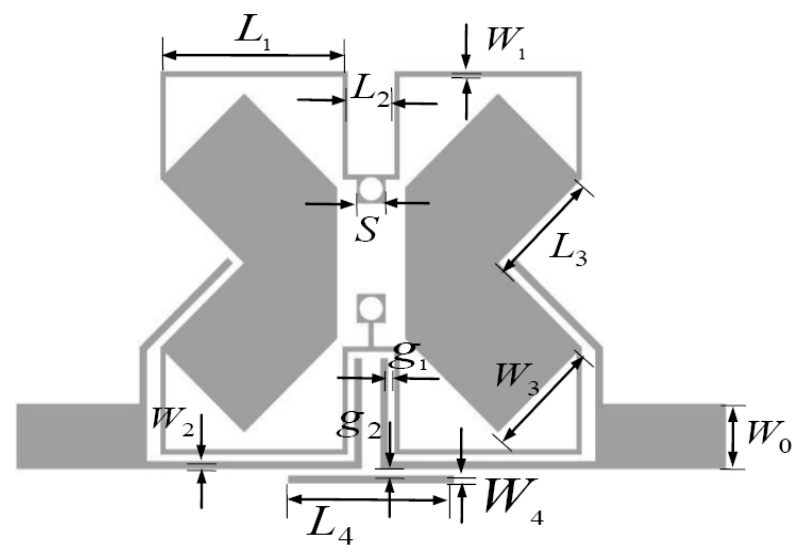

Fig. 4. Layout of final dual-mode filter.

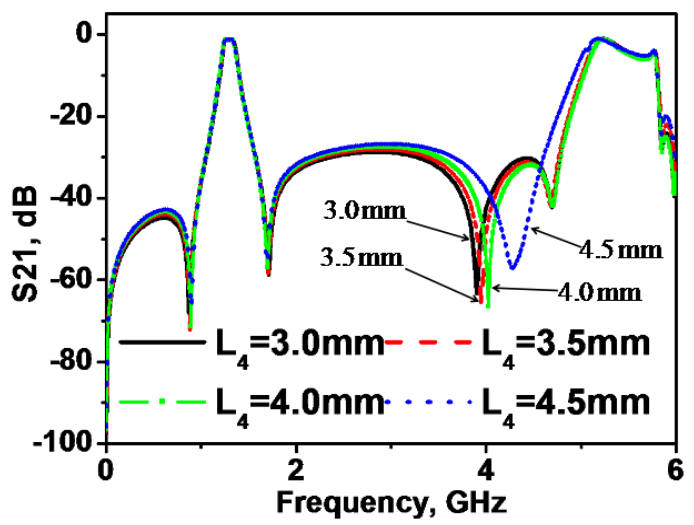

(a) $L_{4}$

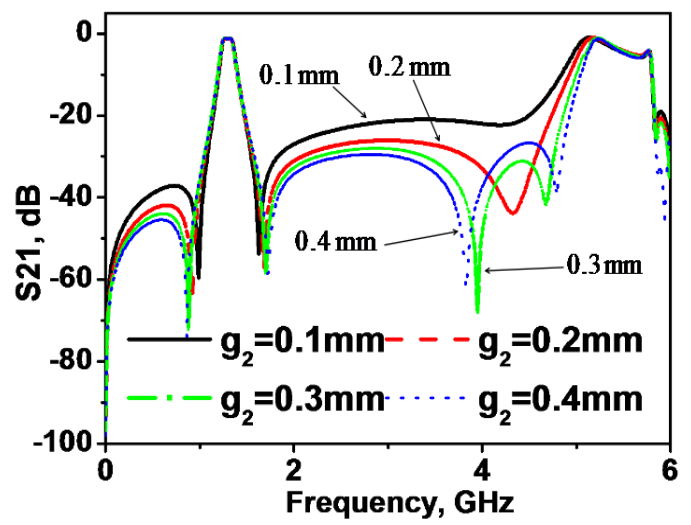

(b) $g_{2}$

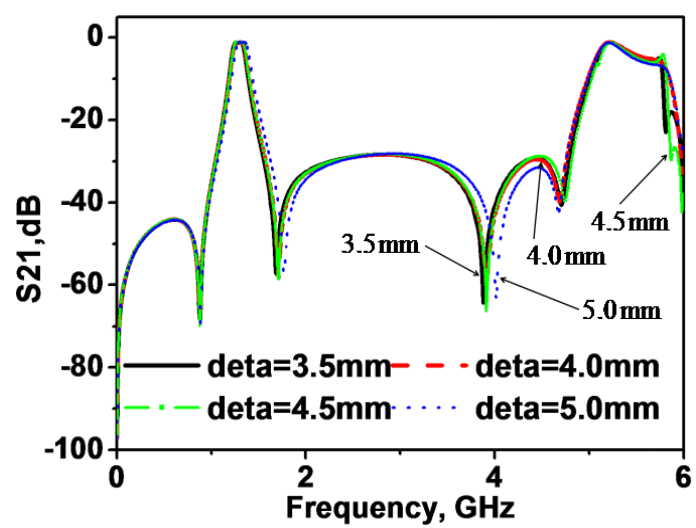

(c) delta

Fig. 5. Simulated S21 of filter for values of $L_{4}, g_{2}$, delta.

$W_{2}$ and $g_{1}$ can affect the results of feed. The best result can be only obtained by designing appropriate feed distance. By adjusting the $W_{2}$ and $g_{1}$ at the same time, we get that $W_{2}=0.3 \mathrm{~mm}$ and $g_{1}=0.1 \mathrm{~mm}$. By simulation, as shown in the Fig. 5(a). We can find that $L_{4}$ can control the location and existence of the third transmission zero. Fig. 5(b) shows that $g_{2}$ affects the location of the transmission zeros and the effect of the feed. $g_{2}$ increases from 1 to $4 \mathrm{~mm}$, the third and forth transmission zeros grow out of nothing. $g_{2}$ also affects the isolation shown in Fig. 5(b). By simulation, choosing $g_{2}=0.3 \mathrm{~mm}$ can reach the best results. Fig. 5 (c) shows the effect of the chamfer adjustment in the filter. The chamfer is equivalent to the load of filter, 
which affects the location of the center frequency of the pass-band and the insertions. The length of the corner cut off (delta) not more than $5 \mathrm{~mm}$ had less effects on filter.

For the design of the filter shown in Fig. 4, whose parameters are given below: $L_{1}=8.2 \mathrm{~mm} ; L_{2}=1 \mathrm{~mm}$; $S=1.2 \mathrm{~mm} ; L_{3}=5 \mathrm{~mm} ; L_{4}=7 \mathrm{~mm} ; \quad W_{1}=0.2 \mathrm{~mm} ; \quad W_{2}=0.3 \mathrm{~mm} ; \quad W_{3}=4.9 \mathrm{~mm} ; \quad W_{4}=0.3 \mathrm{~mm} ;$ $g_{1}=0.1 \mathrm{~mm} ; g_{2}=0.3 \mathrm{~mm}$. The photo of the fabricated filter is shown in Fig. 6 . The overall size of the designed filter is $25 \mathrm{~mm} \times 30 \mathrm{~mm}$, which is a miniaturized structure. Vector network analyzer is used to measure the fabricated filter. Mechanical standards are used to remove the effects of the fixture from the measurement. The application of simulation and measurement can obtain the results shown in Fig. 6 . The simulation results show that the center frequency of the filter is $1.28 \mathrm{GHz}$; the relative bandwidth of $1.3 \mathrm{~dB}$ is $6.2 \%$; the first transmission zero is located at $0.88 \mathrm{GHz}$; the second transmission zero is at $1.70 \mathrm{GHz}$; the third transmission zero is at $3.95 \mathrm{GHz}$; and the fourth transmission zero is at $4.68 \mathrm{GHz}$. High-order resonant frequency is $5.22 \mathrm{GHz}$, which is approximately 4.08 times of the fundamental frequency and similar to the setting of 4.1 times of the fundamental frequency. The $-25 \mathrm{~dB}$ stop-band is $1.52 \mathrm{GHz}-4.81 \mathrm{GHz}$. The measurement results show that the minimum loss of the pass-band is less than $2 \mathrm{~dB}$, and the location of the transmission zeros is slightly different from that of the simulation. These differences may be caused by sampling band width of the simulation software and the actual measurement vector network analyzer and caused by dielectric loss of the substrate.

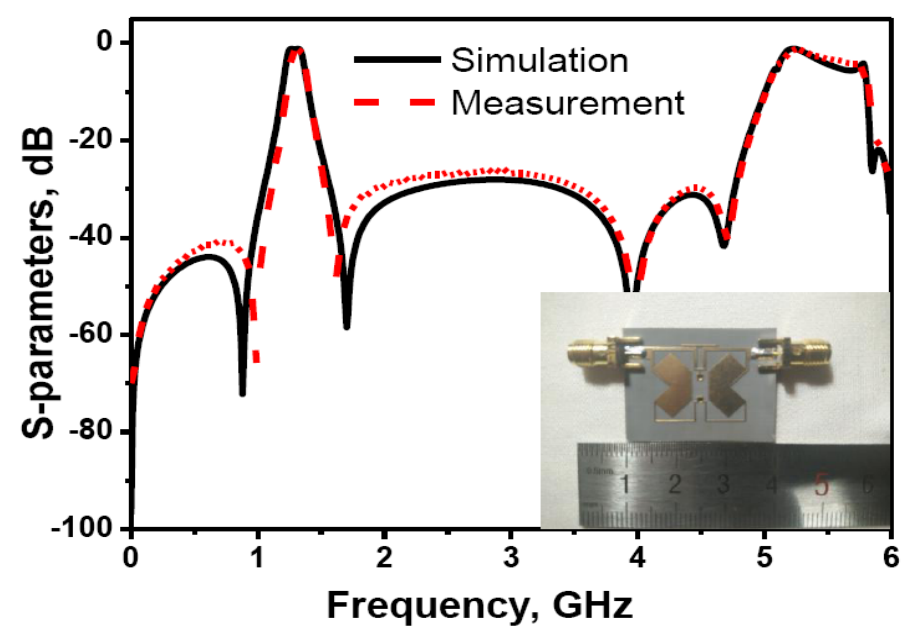

Fig. 6. The simulation and the measurement of the S21 of the filter.

\section{Conclusion}

This paper demonstrates the design of a compact filter with wide stop-band, four controllable transmission zeros, open-ended stub loaded and chamfer lines. And it analyzes the influence of some parameters of the structure on the filter design. Then, an easy design method of the length of feeders is given, which is effective for the design of the feeders. A filter is specially designed, of which the structure is analyzed, to conclude the effects of open-ended stub loaded and the chamfer lines and to obtain the results of full wave analysis simulation. The simulated results are similar to that of the measurement, which proves the reliability of this design. In addition, we can improve the gain of this kind of filers and use this resonant structure in the design of the diplexers.

\section{References}

[1] Yin, Q., Wu, L. S., \& Zhou, L. (2010). Compact dual-band bandpass filter using asymmetrical dual stub- 
loaded open-loops. Journal of Electromagnetic Waves and Applications, 24, 2397-2406.

[2] Lin, X. M. (2010). Design of compact tri-band bandpass filter using $\lambda / 4$ and stub-loaded resonators. Journal of Electromagnetic Waves and Applications, 24, 2029-2035.

[3] Athukorala, L., \& Budimmimir, D. (2009). Compact dual-mode open loop microstrip resonators and filters. IEEE Microw. Wirel. Compon. Lett., 19(11), 698-700.

[4] Sun, J. S., Wu, B., T. Su, Deng, K., \& Liang, C. H. (2012). Wideband dual-mode microstrip filter using short-ended resonator With centrally loaded inductive stub. IEEE Trans. Microw. Theory Tech, 60(12), 3667-3673.

[5] Zhang, X. C., Yu, Z. Y., \& Xu, J. (2008). Design of microstrip dual-mode filters based on source-load coupling. IEEE Microw. Wirel Compon. Lett., 18(10), 677-679.

[6] Wei, C. L., Jia, B. F., Zhu, Z. J., \& Tang, M. C. (2011). Novel trigonal dual-mode filter with controllable transmission zeros. IET Microw. Antennas Propag., 13(5), 1563-1567.

[7] Wei, C. L., Jia, B. F., Zhu, Z. J., \& Tang, M. C. (2011). Hexagonal dual-mode filter with four transmission zeros. Electronics Letters, 47(3).

[8] Chiou, Y. C., Kuo, J. T., \& Cheng, E. (2006). Broadband quasi-Chebyshev bandpass filters with multimode stepped-impedance resonators (SIRs). IEEE Trans. Microw. Theory Techn., 54(8), 3352-3358.

[9] Wang, H., Chu, Q. X., \& Gong, J. Q. (2009). A compact wideband microstrip filter using folded multiple-mode resonator. IEEE Microw. Wireless Compon. Lett., 19(5), 287-289.

[10] Duan, Q., Song, K. J., Chen, F. L., \& Fan, Y. (2015). Compact wide-stopband diplexer using dual mode resonators. Electronics Letters, 51(14), 1085-1087.

[11] Feng, W. J., Gao, X., \& Che, W. Q. (2014). Microstrip diplexer for GSM and WLAN bands using common shorted stubs. Electronics Letters, 50(20), 1486-1488.

[12] Guan, X. H., Yang, F. Q., Liu, H. W., \& Zhu, L. (2014). Compact and high-isolation diplexer using dual-mode stub-loaded resonators. IEEE Microw. Wireless Compon. Lett., 24(6), 385-387.

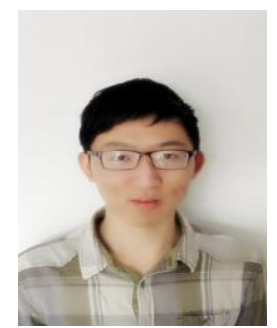

Xue Bing was born in Henan, China, in 1991. He received the B.S. degree in electrical engineering from the University of Electronic Science and Technology of China (UESTC), Chengdu, China, in 2014, and is currently working toward the M.S. degree in the microwave engineering at the Institute of Electronics, Chinese Academy of Sciences, Beijing, China. His research interests include computational eletromagnetics, especially finite element method (FEM), the FEM computing model of Magnetotelluric, the low-frequency electromagnetic wave propagation on the earth, microwave filter and antenna. His current research focuses on the radar filter and antenna.

He has authored two papers in the Chinese refereed journals and one paper in the conference proceeding. They are about the computational eletromagnetics and microwave measurement.

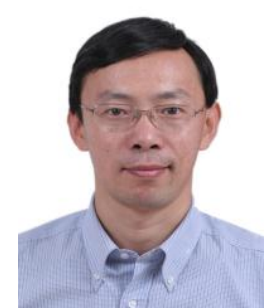

Ji Yicai was born in Shandong, China, in 1974. He received the Ph.D. degree in microwave engineering from Xidian University, Xi'an, China, in 2004. His research interests include computational eletromagnetics, microwave filter and antenna. His current research focuses on the radar antenna.

From 2004 to 2006, he was a Research Assistant with the Xidian University. In 2006, he joined the Institute of Electronics, Chinese Academy of Sciences, Beijing, China, as a Research Associate. Now, he is a Research Professor with the Institute of Electronics, Chinese Academy of Sciences. He has authored or coauthored over 20 papers in the refereed journals and conference proceedings. 